\title{
Oxidative Stress and Cardiovascular Risk: Research Must Persist
}

\author{
Ivan Lozada Martinez ${ }^{1 *}$, Daniela Torres Llinás², Jaine Anaya Rivera ${ }^{2}$, Jennifer \\ Jiménez Valverde ${ }^{2}$ and Milena Castro Berrio ${ }^{2}$
}

${ }^{1}$ Director of Medical-Surgical Research Center, School of Medicine, University of Cartagena, Cartagena Colombia

${ }^{2}$ Researcher, Medical-Surgical Research Center, School of Medicine, University of Cartagena, Cartagena Colombia

*Corresponding author: Ivan David Lozada Martinez, Director of Medical-Surgical Research Center, School of Medicine, University of Cartagena, Cartagena, Colombia

\section{Commentary}

Advances in medicine and experimental biology have supported the definition of concepts related to the process of cellular stress, depending on new discoveries at the molecular level [1]. Oxidative stress is the product of the activity of various metabolic pathways including the mitochondrial electron chain, Nicotinamide Adenine Dinucleotide Phosphate Oxidase (NADPH), nitric oxide synthase, xanthine oxidase, among others [2]. Physiologically, regular levels of reactive oxygen species (ROS) play a fundamental role in cellular and molecular dynamics, however, when there is an imbalance between the production and elimination of these substances (predominant production), a significant stimulus of injury and cell death is generated [3].

Nucleic acids, lipids, and proteins are the main targets of ROS. Levels of oxidative stress have been found to be directly proportional to levels of cellular inflammation [4], therefore, greater persistence in oxidative stress exposure, will cause a state of chronic progressive pathological inflammation. These effects have a considerable impact on the cardiovascular system, since they generate functional damage and morphological alteration in all related structures, precipitating the appearance of cardiovascular diseases. The above is evident in the presentation of subclinical atherosclerosis and insulin resistance, which together with the process of genetic susceptibility and cellular senescence, as well as other mechanisms, give rise to the most prevalent cardiovascular diseases such as endothelial dysfunction, atherosclerosis, hypertrophic cardiomyopathy, myocardial ischemia, myocardial infarction, ischemic/ hemorrhagic stroke, peripheral vascular disease, hypertension, type 2 diabetes mellitus, chronic renal disease, and many more [5]. Therefore, this is and will be for a significant time, a topic of interest in medicine and in other health branches.

Rodríguez-Sánchez, et al. [6] carried out an innovative study in which they evaluated the cardiovascular risk associated with lifelong organic oxidative stress in adults and young people, independently of traditional risk factors, by calculating multiple ROS levels and cardiovascular risk scores, finding that in the young adult population (30 to 50 years) with no history of a cardiovascular event, oxidative stress levels were relatively similar to those with a history of the event, and/or with established coronary artery disease, and therefore the risk of the "healthy" group was high [6]. This may indicate an abysmal underestimation of cardiovascular risk with the use of simple cardiovascular risk scores, which must be taken into account in the clinical practice.

The study of these metabolic pathways has made it possible to determine the impact of aerobic exercise on the dynamics of oxidative stress [7]. Moderate aerobic exercise leads to a transient elevation of ROS, which is compensated with an aggressive discharge of antioxidants, generating an anti-stress environment, and this is observable at the macro level during the improvement in insulin sensitivity, improvement in cardiac function, and regulation of blood pressure by modulation of vascular dynamics [8]. At the micro-level, the findings are more interesting and promising, since it has been specifically determined that it causes an increase in the values of superoxide dismutase- 1 , glutathione reductase,

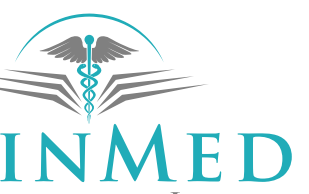

INTERNATIONAL LIBRARY

Citation: Martinez IL, Llinás DT, Rivera JA, Valverde JJ, Berrio MC (2020) Oxidative Stress and Cardiovascular Risk: Research Must Persist. Int Arch Vasc Med 3:007. doi.org/10.23937/2690-3164/1710007 Accepted: December 14, 2020: Published: December 16, 2020

Copyright: (C) 2020 Martinez IL, et al. This is an open-access article distributed under the terms of the Creative Commons Attribution License, which permits unrestricted use, distribution, and reproduction in any medium, provided the original author and source are credited. 
and glutathione peroxidase, and a decrease in inflammatory markers such as interleukin-6, homocysteine, and tumor necrosis factor-alpha [9]. Here is the explicit answer to the need to encourage regular physical exercise and healthy eating habits, and the reason for the degree and number of complications presented in those with an unhealthy lifestyle. It is so important to investigate the role of oxidative stress at the systemic level since the study of these substances has even allowed us to respond to conditions that were incomprehensible such as dementia and vascular depression [10], but that nowadays, we understand a lot of them, knowing already that they are secondary diseases to microvascular lesions to repetition.

Considering then the role of oxidative stress on the pathophysiology of cardiovascular diseases, it is urgent to find new markers to measure the real levels of oxidative stress, in addition to monitoring the conditions already described that induce stress and adaptive mechanisms harmful to cardiovascular level, to establish a personalized risk of suffering a minor or greater cardiovascular event in the short/medium term, and improve the accuracy and quality of diagnostic and therapeutic procedures, as the more complex the disease to be treated, the lower the rate of effectiveness and therapeutic safety, and therefore the higher the risk of morbidity, mortality, and disability.

\section{Financial Support}

None.

\section{Conflict of Interest}

None.

\section{Authors Contribution}

All authors have contributed for this manuscript.

\section{References}

1. Crusio WE, Dong H, RadekeHH, Rezaei N, Xiao J (2020) Frailtyand Cardiovascular Diseases: Research into an Elderly Population. ( $1^{\text {st }}$ edn), Springer, Switzerland.

2. Rubattu S, Forte M, Raffa S (2019) Circulating Leukocytes and Oxidative Stress in CardiovascularDiseases: A State of the Art. Oxid Med Cell Longev 2019: 2650429.

3. Finkel T (2011) Signal transduction by reactive oxygen species. J Cell Biol 194: 7-15.

4. Martin-Ventura JL, Rodrigues-Diez R, Martinez-Lopez D, Salaices M, Blanco-Colio LM, et al. (2017) Oxidative Stress in Human Atherothrombosis: Sources, Markers and Therapeutic Targets. Int J Mol Sci 18: 2315.

5. Ndrepepa G (2019) Myeloperoxidase - A bridge linking inflammation and oxidative stress with cardiovascular disease. Clin Chim 493: 36-51.

6. Rodríguez-Sánchez E, Navarro-García JA, Aceves-Ripoll J, González-Lafuente L, Corbacho-Alonso N, et al. (2019) Lifetime cardiovascular risk is associated with a multimarker score of systemic oxidative status in young adults independently of traditional risk factors. Transl Res 212: 54-66.

7. Luca M, Luca A (2019) Oxidative Stress-Related Endothelial Damage in Vascular Depression and Vascular Cognitive Impairment: Beneficial Effects of Aerobic Physical Exercise. Oxid Med Cell Longev 2019: 8067045.

8. Ristow $\mathrm{M}$, Zarse $\mathrm{K}$, Oberbach $\mathrm{A}$, Klöting $\mathrm{N}$, Birringer $\mathrm{M}$, et al. (2009) Antioxidants prevent health-promoting effects of physical exercise in humans. Proc Natl Acad Sci USA 106: 8665-8670.

9. Koloverou E, Tambalis K, Panagiotakos DB, Georgousopoulou E, Chrysohoou C, et al. (2018) Moderate physical activity reduces 10 -year diabetes incidence: The mediating role of oxidative stress biomarkers. Int $\mathrm{J}$ Public Health 63: 297-305.

10. Diniz BS, Butters BA, Albert SM, Dew MA, Reynolds CF (2013) Late-life depression and risk of vascular dementiaand Alzheimer's disease: Systematic review and meta-analysis of community-based cohort studies. Br J Psychiatry 202: 329-335. 